\title{
Entre tensions textuelles et spectaculaires : Écrire le masque et la danse dans Love's Labour's Lost
}

Samuel Cuisinier-Delorme

\section{(2) OpenEdition \\ Journals}

Édition électronique

URL : http://journals.openedition.org/shakespeare/2935

DOI : 10.4000/shakespeare.2935

ISSN : 2271-6424

Éditeur

Société Française Shakespeare

Référence électronique

Samuel Cuisinier-Delorme, «Entre tensions textuelles et spectaculaires : Écrire le masque et la danse dans Love's Labour's Lost », Actes des congrès de la Société française Shakespeare [En ligne], 32 | 2015, mis en ligne le 10 mars 2015, consulté le 22 juillet 2020. URL : http://journals.openedition.org/ shakespeare/2935 ; DOI : https://doi.org/10.4000/shakespeare.2935

Ce document a été généré automatiquement le 22 juillet 2020.

(c) SFS 


\title{
Entre tensions textuelles et spectaculaires : Écrire le masque et la danse dans Love's Labour's Lost
}

\author{
Samuel Cuisinier-Delorme
}

1 Love's Labour's Lost est l'une des pièces de Shakespeare où la dimension chorégraphique est la plus prégnante, bien qu'aucune danse n'y soit exécutée. Un réseau lexical abondant ${ }^{1}$ traverse la pièce, complété par une série d'allusions, de métaphores, de commentaires plus ou moins en lien avec le champ sémantique de la chorégraphie. Le metteur en scène Darko Tresnja souligne d'ailleurs que «Love's Labour's Lost est presque chorégraphiée sur le papier ${ }^{2}$.» Dans cette pièce méta-dramatique par excellence, le spectaculaire, embrassant théâtre et danse, y est longuement discuté. La danse a alors toute sa place dans le discours puisqu'« elle n'est pas non plus sans rapport avec la rhétorique ${ }^{3}$, comme le remarque Lucien de Samosate dans son Éloge de la danse.

\section{Les usages de la danse dans le théâtre de Shakespeare}

2 En préambule, il convient de rappeler les usages chorégraphiques dans le théâtre de Shakespeare. La danse apparaît à trois niveaux du jeu. Il s'agit tout d'abord d'un élément de mise en scène: les didascalies, externes et internes, prévoient son interprétation et son exécution sur le plateau. Elle offre, dans sa forme élémentaire, un intermède distrayant pour le spectateur. Intégrée de manière plus complexe, tout en conservant sa fonction divertissante, elle permet des avancées narratives (par exemple, dans la scène de bal de Romeo and Juliet, l'intrigue est intrinsèquement liée à la danse). Néanmoins, son absence scénique ne signe pas sa disparition définitive: l'art de Terpsichore ressurgit en effet de manière linguistique. L'anatomie chorégraphiée est ainsi lexicalisée. Évacuée de la représentation, la danse connaît une renaissance en contaminant le texte par des mentions régulières. Enfin, la chorégraphie participe à un mouvement structurel et symbolique : la danse est cosmique, mais la variation du motif 
tend parfois vers le tragique. Elle revêt, dans le répertoire shakespearien et dans la culture de la Renaissance, une dimension idéologique. Elle véhicule une représentation de la société et du monde ; ses multiples usages en attestent. Toutefois, sa dimension technique est toujours occultée: Shakespeare n'impose jamais de forme chorégraphique et il n'en décrit aucune. La dimension esthétique du geste est "délaissée » au profit de sa fonction expressive et rhétorique. Au sens premier du verbe, c'est-à-dire "représenter (une notion abstraite) sous une forme matérielle et visible $^{4}$ ", la danse incarne un discours parfois proche de la philosophie.

3 L'analyse générique et chronologique du motif chorégraphique dans le théâtre de Shakespeare confirme que la danse est, pour citer à nouveau Lucien, « loin d'être un de ces arts faciles qu'on apprend sans mal5.»Le dramaturge fait montre d'une connaissance de plus en plus subtile et habile de l'art chorégraphique, et son intégration se fait de plus en plus complexe. Dans les pièces historiques, écrites essentiellement au début de sa carrière, la danse est uniquement un support d'images métaphoriques, lesquelles permettent notamment de prolonger la théorie du corps politique (body politic) qui dessine des analogies entre le corps humain et le pouvoir. Néanmoins, les représentations du pouvoir par la danse dans le théâtre de Shakespeare soulignent essentiellement la discorde du royaume et donnent généralement l'image d'un monarque inexpérimenté sur les plans politique et militaire : le dramaturge renverse l'idéologie selon laquelle un homme éduqué est un homme qui sait danser. La scène de bal de Romeo and Juliet (I.v) est la première à spécifier l'exécution d'une danse sur le plateau ${ }^{6}$. Les premières comédies, dans la continuité des pièces historiques, ne comportent aucun passage chorégraphié, alors que les suivantes - A Midsummer Night's Dream, Much Ado about Nothing, As You Like It - se concluent, quant à elles, par une danse afin de rappeler la réconciliation des couples et célébrer leurs noces. Elle vient mettre un point final à la suite de péripéties ayant troublé l'ordre initial. On remarque donc que l'écriture de Love's Labour's Lost correspond, sur le plan chorégraphique, à un tournant de l'œuvre de Shakespeare : cette comédie apparaît comme le point de départ d'une intégration croissante de la danse. L'art chorégraphique est omniprésent dans cette pièce tout comme dans A Midsummer Night's Dream, vraisemblablement écrite à la même période, mais son incorporation est diamétralement opposée dans l'une et l'autre de ces deux pièces. Shakespeare calque la structure diégétique de la première sur une forme chorégraphique ${ }^{7}$, sans qu'aucune danse ne soit exécutée. À l'inverse, $A$ Midsummer Night's Dream est une célébration, voire une consécration, de la danse : les artisans, les nobles et les fées embrassent l'art de Terpsichore, unissant macrocosme et microcosme à travers des rondes et d'autres formes chorégraphiques.

4 Ainsi, dans Love's Labour's Lost, l'essence même de la danse est rejetée hors cadre scénique et diégétique : l'exécution chorégraphique est occultée de la scénographie. Néanmoins, la description des déplacements et de l'occupation de l'espace au sein des pièces est une forme chorégraphique envisagée de manière lexicale. Le texte shakespearien s'écrit alors comme une série d'hypotyposes de la danse articulées autour de réseaux sémantiques formés par les répliques et les didascalies.

\section{Ecrire la danse : le « masque verbal»}

La scène finale de la pièce (V.ii) participe à une expérience iconique et met en œuvre, selon l'expression empruntée à Anne Ubersfeld, des «matrices textuelles de 
représentativité8.» Par sa structure, le dénouement de la comédie s'apparente à un masque, spectacle de cour intégrant théâtre, danse et musique dans une scénographie complexe.

Dans les pièces de Shakespeare, la forme du "masque " n'est globalement pas jacobéenne mais élisabéthaine. La chronologie explique cet état de fait : la plupart des pièces où le terme est mentionné ont été écrites, sous le règne d'Élisabeth I⿳亠े冖e entre 1590 et 1600 ; il faut alors le comprendre comme mascarade ou momerie, c'est-à-dire comme une danse masquée. Le divertissement se compose essentiellement d'une entrée suivie d'une danse :

The word always refers to an Elizabethan type of masque, consisting of an entrance and a dance. [...] Not only is the linguistic usage constant, but Shakespeare has a tendency to couple 'masque' with 'revel' or 'dance' in a general allusion to festivity, destroying any particularity in the conception of a masque?.

Les pièces ayant recours à ces formes chorégraphiées les utilisent non pas comme esthétique de la scénographie, mais comme ressort dramatique. Elles permettent une avancée de l'histoire : la révélation est au cœur de ces danses et fournit généralement des informations que seul le déguisement peut divulguer. Comme le souligne Jan Kott dans The Gender of Rosalind, «le déguisement voile mais surtout dévoile et met à nu ${ }^{10}$. »

7 La dernière scène de Love's Labour's Lost, (V.ii) met en jeu des masques en tant qu'accessoires, mais les personnages ne dansent pas, malgré la musique :

ROSALINE. Play music, then! [Music plays] Nay, you must do it soon. Not yet? No dance!

$\left(\right.$ V.ii.211-12) ${ }^{11}$

Les hommes ont renoncé aux plaisirs frivoles sans parvenir à respecter leur vœu, et les femmes ne sont pas prêtes à se laisser courtiser par eux, ou du moins le laissent-elles croire pour que les séducteurs poursuivent leur dessein. La scène est construite autour de situations burlesques mises en scène par les personnages eux-mêmes :

ROSALINE. Which of the vizards was it that you wore? BEROWNE. Where, when, what vizard? Why demand you this? ROSALINE. There, then, that vizard, that superfluous case That hid the worse and showed the better face.

KING. [Aside] We are descried. They'll mock us now downright. DUMAINE. [Aside] Let us confess and turn it to a jest.

(V.ii.385-90)

8 Le public, dans la confidence de la supercherie de la scène, est complice de la mauvaise foi des personnages. Rosaline se défausse : elle critique l'accessoire alors qu'elle a, elle aussi, utilisé un masque. Berowne et Ferdinand font quant à eux part de leur étonnement et prétendent ne pas en avoir porté. Même démasqué, chaque personnage s'affaire à tenir un rôle. Prises à leur propre jeu, les femmes feignent donc ne pas reconnaître les hommes qui leur font la cour, déguisés en Moscovites $^{12}$ :

ROSALINE. What would these strangers? Know their minds, Boyet.

If they do speak our language, 'tis our will

That some plain man recount their purposes.

Know what they would.

(V.ii.175-79) 
Les deux groupes se confrontent tout au long de cette scène finale sous la forme de micro-séquences qui reprennent la structure du masque Tudor. Celui-ci se composait généralement de l'entrée de personnages masqués et costumés qui arrivaient de manière impromptue lors d'un banquet ${ }^{13}$. Accompagnés d'un Présentateur, ils exécutaient une danse avant de se mêler aux convives. L'entrée était parfois précédée d'un prologue pour expliquer la venue de ces invités inattendus. Shakespeare détourne ici certains codes de cette forme spectaculaire :

\begin{tabular}{|c|c|}
\hline $1^{\mathrm{er}}$ masque & $\begin{array}{l}\text { Les dames se masquent } \\
\text { "The trumpet sounds. Be masked; the maskers come. » (157) } \\
\text { Entrée des messieurs } \\
\text { Enter Blackamoors with music [Moth,] the Boy with a speech, and the rest of the lords } \\
\text { disguised [as Russians]. (157.2) } \\
\text { Echanges en couple / apartés: } \\
\text { - Rosaline/King (203-229) They converse apart (229.1) } \\
\text { - Berowne/Princess (230-237) They converse apart (237.1) } \\
\text { - Dumaine/Maria (238-241) They converse apart (241.1) } \\
\text { - Katherine/Longaville (242-255) They converse apart (255.1) } \\
\text { Sortie des messieurs } \\
\text { Exeunt [the King, lords and attendants] (264.1) }\end{array}$ \\
\hline Intermède & $\begin{array}{l}\text { Discussion des dames (265-309) } \\
\text { Sortie des dames } \\
\text { Exeunt [the Princess and ladies] (309.1) } \\
\text { Entrée des messieurs } \\
\text { Enter the KING and the rest [BEROWNE, LONGAVILLE, and DUMAINE, as themselves] } \\
(309.2-3) \\
\text { Discussion des messieurs (310-336) }\end{array}$ \\
\hline $2^{\mathrm{e}}$ masque & $\begin{array}{l}\text { Entrée des dames } \\
\text { Enter the ladies [ the PRINCESS, ROSALINE, MARIA, and KATHERINE, with BOYET] } \\
(336.1-2) \\
\text { Échanges en couple: } \\
\text { - King/Princess (339-363) } \\
\text { - Rosaline/Berowne (364-430) } \\
\text { - King/Princess (431-442) } \\
\text { - Tous les quatre (443-483) }\end{array}$ \\
\hline Intermède & $\begin{array}{l}\text { Spectacle des Neuf Preux (484-690) } \\
\text { Interruption du spectacle (691) }\end{array}$ \\
\hline
\end{tabular}




\begin{tabular}{|l|l|}
\hline & Discussion commune (691-760) \\
& $\begin{array}{l}\text { Échanges en couple / apartés : } \\
\text { - King/Princess (761-790) They converse apart (790.1) } \\
\text { - Dumaine/Katherine (791-800) They converse apart (800.1) } \\
\text { - Maria/Longaville (801-804) They converse apart (804.1) } \\
\text { Danse } \\
\text { collective }\end{array}$ \\
& - Berowne/Rosaline (805-839) \\
\hline Intermède & Chants (857-894) \\
\hline Sortie & Sortie des messieurs et des dames (896.1) \\
\hline
\end{tabular}

10 La scène repose sur des oppositions symétriques : les groupes (hommes/femmes) et les couples, scènes d'ensemble et apartés, entrées et sorties... Le groupe est une matrice autour de laquelle gravitent les couples : ceux-ci sont contraints de respecter l'action collective. On remarquera que l'initiative est toujours celle d'un groupe, jamais celle d'un individu. Si c'est le cas, le particulier défend toujours une position collective. Les déplacements se limitent à la didascalie "They converse apart »; la préposition impose une mise à l'écart, excluant de fait le spectateur du reste de la conversation.

11 La première entrée des gentilshommes marque le début d'un masque de forme Tudor, se présentant comme une arrivée impromptue de personnages masqués qui se mêlent aux convives. À sa suite, un intermède permet des commentaires sur le divertissement, comme si le spectateur était convié à entrer dans les coulisses. Les seigneurs ne sont plus déguisés (Enter... as themselves). Ce masque est suivi d'un second, initié cette fois-ci par les dames accompagnées de Boyet, dans l'emploi du Présentateur. Les masques sont tombés, et cette fois-ci, les couples sont réunis.

12 Le spectacle des Neuf Preux est un intermède mis en abyme : il est enchâssé dans une séquence qui relève d'un divertissement plus ou moins improvisé. Interrompue, la représentation laisse place à la réunion des quatre couples. Toutefois, celle-ci est empêchée par les chants puis par la sortie, de part et d'autre de la scène, de chacun des groupes. Cette dernière partie forme un «triptyque » sous la configuration ensemble / couple / ensemble et rappelle les danses collectives des masques jacobéens, plus tardifs. D'un point de vue historique, ces trois micro-séquences, assimilables à des " masques verbaux ", montrent la fluctuation d'une forme qui n'est pas encore fixée mais qui, en cette fin de XVI ${ }^{e}$ siècle, porte en germe la structure du masque jacobéen.

13 Le spectacle des Neuf Preux ${ }^{14}$ contient lui aussi une forte "charge chorégraphique ». Anthony Dull propose à Holofernes ses services de danseur et/ou de musicien pour la parade que le roi veut offrir à la princesse :

HOLOFERNES. Allons! We will employ thee.

DULL. I'll make one in a dance, or so; or I will play on the tabor to the Worthies, and let them dance the hay.

HOLOFERNES. Most dull, honest Dull! To our sport, away! Exeunt

(V.i.122-25)

L'enchâssement de ce spectacle, dont le thème était très répandu à la Renaissance ${ }^{15}$, a pour but de divertir les femmes et offrir aux hommes l'opportunité de se montrer sous 
leur meilleur jour. Paradoxalement, ces derniers seront masqués et déguisés. Leur tentative reste vaine, et le spectacle s'avère parodique et grotesque (d'autant qu'ils ne sont que cinq pour incarner neuf personnages). La représentation, menacée par les altercations opposant Armado à Dull, ne sera pas menée à son terme.

Dull propose, comme nous l'avons vu, ses services de danseur ou de musicien pour le spectacle. L'antonomase laisse deviner les capacités artistiques du personnage, selon la polysémie de l'adjectif dull :

1. Not quick in intelligence or mental perception; slow of understanding;

2. Wanting sensibility or keenness of perception in the bodily senses and feelings [...]. In dialect use, esp. Hard of hearing, deaf.

3. Slow in motion or action ${ }^{16}$.

L'onomastique indique donc clairement que le personnage n'est pas apte à participer à la représentation. Son manque de discernement (sens 1) l'amène à envisager d'exécuter une farandole, danse médiévale paysanne en décalage avec la finalité du spectacle des Neuf Preux : cette forme chorégraphique ne sied pas aux héros mythiques et n'est pas propice à la séduction. Au contraire, une danse militaire, glorifiant les personnages, serait à prescrire ici. Dans son dictionnaire de la danse, G. Desrat souligne la "rapidité proverbiale ${ }^{17}$ » de la farandole qui contraste ici avec la lenteur supposée de Dull (sens 3). Ce manque de vivacité n'est pas non plus à son avantage lorsqu'il suggère de jouer du tambourin pour accompagner la farandole: alliée à des problèmes de rapidité d'exécution, la surdité (sens dialectal de dull) du gendarme risque de rendre le rythme discordant. Enfin, son incapacité à percevoir des sensations corporelles (sens 2) ferait de lui un piètre danseur. En d'autres termes, la kinesthésie (définie comme la « forme de sensibilité qui, indépendamment de la vue et du toucher, renseigne d'une manière spécifique sur la position et les déplacements des différentes parties du corps $\left.{ }^{18} »\right)$ est nécessaire dans l'appréhension du geste chorégraphié, et Dull semble en être dépourvu. Perplexe et dépassé par la situation, ce dernier participe sans le vouloir au comique et au grotesque de la scène :

HOLOFERNES. Via, goodman Dull! Thou hast spoken no word all this while. DULL. Nor understood none neither, sir.

(V.i.120-22)

\section{De l'absence de danse, ou comment expliquer l'épilogue}

Afin d'analyser cette scène dans son ensemble sur le plan chorégraphique, il convient désormais d'étudier l'absence de danse à la fin de la pièce, cet «épilogue chorégraphique » attendu, mais manquant.

La symbolique de la danse dans le théâtre de Shakespeare s'explique en grande partie par le genre des pièces. Certaines tendances se dégagent en considérant les quatre grandes catégories du répertoire shakespearien (pièces historiques, tragédies, comédies et romances tardives), comme le résume Nancy Isenberg :

In the comedies, dancing typically figures harmony and order restored, in the tragedies it tends to signal ironically the opposite. In Shakespeare's later plays, mostly romances, the themes of order and discord are woven together 
through the verbal and performative discourses of dance. The history plays never call for dancing on the stage, nor do the Roman plays, with the one exception of the Egyptian Bacchanal ${ }^{19}$. suivantes - comme A Midsummer Night's Dream, Much Ado about Nothing, As You Like It - se concluent par une danse afin de rappeler la réconciliation des couples et de célébrer leurs noces ${ }^{20}$. Celle-ci répond donc aux codes de la comédie, dans laquelle le dénouement «débouche presque toujours sur une conclusion optimiste (mariage, réconciliation, reconnaissance $)^{21}$. " Elle vient mettre un point final, de manière festive, à la suite de péripéties ayant troublé l'ordre initial :

Shakespeare's comedies often end with a dance, we have been assured, because they move from confusion to order, from misfortune to prosperity, and dancing is an image of concord that celebrates this festive resolution ${ }^{22}$.

Le schéma des comédies repose en partie sur le désordre amoureux. La réconciliation qui s'ensuit fait office de tableau harmonieux, objet de quête aussitôt que l'équilibre des sentiments a été perturbé. Le dénouement intègre trois modi operandi différents pour expliciter la réunion des personnages, avant leur sortie de scène :

- l'expression verbale (The Taming of the Shrew);

- le départ pour un banquet (The Comedy of Errors, The Two Gentlemen of Verona, A Midsummer Night's Dream) : "[The communal departure for a banquet or feast, the traditional celebration of unity and concord,] embraces not only the lovers, but all the other characters who can share the celebration ${ }^{23}$. »

- l'exécution d'une danse (A Midsummer Night's Dream, Much Ado about Nothing, As You Like It), dont sont privés les scélérats. Ces personnages discordants sont en effet exclus du schème harmonieux que symbolise l'acte dansé.

Ces trois modes opératoires peuvent être combinés. Toutefois, le dénouement de Love's Labour's Lost fait exception et ne répond à aucune de ces catégories à cause du report de la célébration des noces douze mois et un jour plus tard. La danse, en toute logique, ne peut avoir lieu. Dans son étude de la pièce, Harley Granville-Barker suggère pourtant que le chant allégorique qui clôt la pièce est nécessairement accompagné d'une danse :

But this is not the end, for the end must not be melancholy. The countryfolk have yet to sing and dance their antic, a little crowd of them, dressed to match

daisies pied and violets blue,

And lady-smocks, all silver-white,

And cuckoo-buds of yellow hue...

The comedy of affectations comes to its full close upon notes of pastoral freshness and simplicity ${ }^{24}$.

Alors que Shakespeare a pris soin de n'aborder la danse que par le langage dans cette comédie, pourquoi y aurait-il nécessairement une chorégraphie à la fin de la pièce ? Aucun élément textuel et/ou contextuel ne permet de confirmer ou d'infirmer l'hypothèse de Granville-Barker, qui est mentionnée (mais n'est pas tranchée) dans l'étude d'Alan Brissenden ${ }^{25}$. Ce dernier remarque néanmoins que «le monde de la pièce déborde de la scène et embrasse le nôtre, celui du public ${ }^{26}$. » Dès lors, comme il était de tradition de danser avec le public à la fin d'une représentation, ce moment de «communion chorégraphiée » entre acteurs et spectateurs pourrait célébrer cette 
union rejetée hors de la diégèse, et symboliserait l'harmonie non atteinte dans la fiction.

\section{Conclusion}

Love's Labour's Lost n'a de cesse de célébrer la danse par les paroles, sans que jamais un pas ne soit esquissé. Le texte se donne à lire comme un espace chorégraphique et chorégraphié, un préambule méta-chorégraphique dans lequel Shakespeare ouvre la voie à l'inclusion grandissante du motif dansé. Toutefois, lorsqu'il aborde le registre tragique au tournant du XVII siècle, les danses se font plus rares. Elles sont exécutées dans la première moitié des drames, avant que l'acmé ne fasse profondément et irrémédiablement voler en éclat la fausse harmonie qui émane des personnages.

Le dramaturge affine son utilisation du motif jusqu'à l'inverser dans les romances tardives. Il y remet alors en question la notion d'harmonie à laquelle la danse a toujours été associée. À bien y regarder, en privant le spectateur d'une danse sur le plateau lors $\mathrm{du}$ dénouement de Love's Labour's Lost, Shakespeare ne suggérerait-il pas un renversement précoce du symbole chorégraphique, signalant par là même un détournement bien plus profond des codes de la comédie?

\section{NOTES}

1. On ne compte pas moins de douze occurrences du terme dance, et la basse-danse (V.ii), le branle (III.i), la gigue (III.i et IV.iii) et la canarie (III.i) sont mentionnées.

2. Ce propos de Darko Tresnjak, metteur en scène, provient du dossier de présentation de Titus Andronicus pour le Festival Stratford (Canada) en 2011 : «Love's Labour's Lost and Titus Andronicus are practically choreographed on the page - precisely and intricately plotted dances of love and death, rounds of seduction and revenge, respectively. "

3. Lucien, Éloge de la danse, traduit du grec par Claude Terreaux, Paris : Arléa, 2007, p. 22-23.

4. «Incarner ", Centre National de Ressources Textuelles et Lexicales, CNRTL, en ligne, consulté le 27 janvier 2015 : <http://www.cnrtl.fr/definition/incarner>.

5. Lucien, op. cit., p. 22.

6. William Shakespeare, Romeo and Juliet, The Arden Shakespeare, Second Series, ed. Brian Gibbons, Londres : Thomson Learning, 1980, p. 115 : « Music plays and they dance.» (I.v.26.1).

7. Voir Samuel Cuisinier-Delorme, «L'écriture chorégraphique comme matrice textuelle de Love's Labour's Lost » in Love's Labour's Lost de Shakespeare, ou l'art de séduire, Cycnos, volume $31, \mathrm{n}^{\circ} 1$, éd. Christian Gutleben, Paris : L'Harmattan, 2015, p. 95-110.

8. Anne Ubersfeld, Lire le théâtre I, Paris : Belin Lettres Sup, 1996, p. 16-17.

9. Suzanne Gossett, "The Term 'Masque' in Shakespeare and Fletcher, and The Coxcomb", Studies in English Literature, 1500-1900, Rice University, Vol. 13, No. 2, Elizabethan and Jacobean Drama (Spring, 1974), p. 289-90.

10. Jan Kott, The Gender of Rosalind, Interpretations: Shakespeare, Büchner, Gautier, Northwestern University Press, 1992, p. 17: «Disguise not only covers but also uncovers and bares » (ma traduction). 
11. William Shakespeare, Love's Labour's Lost, The New Cambridge Shakespeare, ed. William C. Carroll, Cambridge : CUP, 2009.

12. Le costume renvoie au domaine chorégraphique, dans le sens où la moscovite est le «nom d'une ancienne contre-danse française, sorte de pot-pourri composé de figures empruntées à diverses danses et exécutée par un nombre de couples ad libitum. » Voir G. Desrat, " Moscovite », Dictionnaire de la Danse, historique, théorique, pratique et bibliographique; depuis l'origine de la danse jusqu'à nos jours, Charleston : Nabu Press, 2010 [1895], p. 249-50.

13. Voir Henry VIII (I.iv) ou Romeo and Juliet (I.v) pour des exemples de masques.

14. Motif symbolisant les valeurs et vertus du parfait chevalier, composé de trois héros païens (Hector, Alexandre le Grand, Jules César), trois personnages de l'Ancien Testament (Josué, Judas Macchabée, le roi David) et trois héros chrétiens (Godefroi de Bouillon, le roi Arthur et Charlemagne).

15. «La popularité des Neuf Preux se traduit par une abondante iconographie, ils sont présents sur les tapisseries, les peintures murales, les gravures, les cartes à jouer jusqu'à la fin du $\mathrm{XVI}^{\mathrm{e}}$ siècle. » (Sophie Cassages-Brouquet, « Penthésilée, reine des Amazones et Preuse, une image de la femme guerrière à la fin du Moyen Âge ». Source en ligne, consultée le 27 janvier 2015 : http:// clio.revues.org/1400?\&id=1400 )

16. « Dull », OED, en ligne, consulté le 27 janvier 2015 : <http://www.oed.com/view/Entry/58304>. 17. G. Desrat, « Farandole », op. cit., p. 139.

18. "Kinesthésie ", Centre National de Ressources Textuelles et Lexicales, CNRTL, en ligne, consulté le 27 janvier 2015 : <http://www.cnrtl.fr/definition/kinesthésie>.

19. Nancy Isenberg, "Dancing with the Stars in Antony and Cleopatra », in Questioning Bodies in Shakespeare's Rome, eds. Maria Del Sapio Garbero, Nancy Isenberg,

20. Bien que Twelfth Night compte deux mariages, aucune danse n'accompagne le dénouement de cette comédie. Cette absence marque-t-elle une impossibilité d'atteindre l'harmonie? Shakespeare réutilise ici les codes et procédés de ses pièces précédentes, mais il introduit dans sa pièce le doute et la mélancolie.

21. Patrice Pavis, « Comédie », Dictionnaire du théâtre, Paris : Armand Collin, 2002, p. 52.

22. Skiles Howard, The Politics of Courtly Dancing in Early Modern England, University of Massachusetts Press, 1998, p. 69.

23. Ibid., p. 138.

24. Harley Granville-Barker, Granville-Barker's Prefaces to Shakespeare, Love's Labour's Lost, Londres : Nick Hern Books, 1993, p. 47.

25. Voir Alan Brissenden, Shakespeare and the Dance, Alton : Dance Books Ltd, 1981, p. 37 et p. 41. 26. Alan Brissenden, op. cit., p. 37: « The world of the play overflows the stage and embraces ours, the audience's. » (ma traduction)

\section{RÉSUMÉS}

Cet article propose dans un premier temps l'étude des différents usages de la danse dans le théâtre de Shakespeare (de sa représentation sur scène à sa valeur symbolique) puis une lecture de la scène finale (V.ii) de Love's Labour's Lost à travers le prisme de la danse. Si aucune chorégraphie n'est exécutée sur le plateau dans cette comédie, de nombreuses références explicites et implicites à l'art de Terpsichore traversent le texte. Ainsi, le but de cet article est de 
reconsidérer la dernière scène de la pièce à la lumière du masque, divertissement de cour dont la structure fournit un cadre diégétique à cette scène de dénouement. Alors que Shakespeare a pris soin d'aborder l'art chorégraphique uniquement par le langage dans Love's Labour's Lost, il convient également de s'interroger sur l'absence de danse en conclusion de la pièce, «épilogue chorégraphique » symbolique et commun à un certain nombre de comédies, mais dont celle-ci est privée.

This article is first a study of the various uses of the dance in Shakespeare's drama (from its representation on stage to its symbolic value) before reading the last scene of Love's Labour's Lost (V.ii) through the prism of the dance. Even though no form of dancing is actually performed on stage in Love's Labour's Lost, the art of Terpsichore is referred to on several occasions through numerous explicit or implicit references in the text. So, this article aims at reconsidering the last scene in the play in the perspective of the masque, a court entertainment whose structure serves as a diegetic frame to the winding up of the play. Even though Shakespeare only tackles choreography through language in Love's Labour's Lost, it is intriguing to find no dance, no choreographic epilogue as a conclusion to the play while this symbolic moment is common to many other comedies except in this particular one.

INDEX

Mots-clés : danse, intersémioticité, masque, Peines d'amour perdues

Keywords : dance, intersemioticity, Love's Labour's Lost, masque

\section{AUTEUR}

\section{SAMUEL CUISINIER-DELORME}

CERHAC, Université Blaise Pascal, Clermont-Ferrand 